\title{
INHALT
}

\section{VORWORT DER AUTORIN}

$1 \quad$ Anlass der Rettungsgrabungen auf der Marktstätte und in der Brotlaube _.............. 14

$2 \quad$ Kurze historische Einführung: Von den Anfängen bis zur frühen Neuzeit …..............14

3 Marktstätte in Schrift- und Bildquellen..

4 Archäologischer Forschungsstand zu Beginn der Ausgrabungen und Fragestellung ....24

5 Durchführung der Grabungen

6 Auswertung der Grabungsdokumentation

7 Perioden und ihre Datierung

2 GRABUNGSBEFUNDE UND REKONSTRUKTION

Natürlicher Untergrund

Obere (westliche) Marktstätte

Untere (östliche) Marktstätte.

Brotlaube

Grabungsbefunde auf der oberen (westlichen) Marktstätte

Periode I: Älteste Befunde in der Flachwasserzone.

Periode II-IIIb: Auffüllung der Flachwasserzone, Holzkonstruktionen und verschiedene Siedlungsaktivitäten.

Periode IV-IVe: Schwellrahmenbau auf Pfostenfundamenten.

Periode $V$-Va: Kleinbau mit Flechtwerkwänden

Periode VI-VIa.1: Schwellenbau und hafenseitiger Steinbau

Periode VII-VIIa.5: Zweiteiliger Großbau; westlicher Schwellenbau auf

Unterlegsteinen und östlicher Schwellenbau auf mörtelgebundener Steinlage _................73

Periode VIII-VIIIa.1: Hafenseitiger Steinbau mit angebautem Schwellenbau ._................89

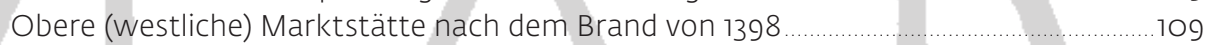

Grabungsbefunde westlich der Hausbebauung (zweite Hälfte 12. Jh. bis zum Brand von 1398)

Grabungsbefunde auf der unteren (östlichen) Marktstätte

Anlage eines Hafenbeckens.

Zuschüttung des Hafenbeckens nach dem Brand von 1398, Erweiterung der Marktstätte zum See hin und Nutzung..

Grabungsbefunde in der Brotlaube

Älteste Befunde: Ablagerungen auf dem Seegrund

Periode II: Bau eines Holz-Erddammes.

Periode lla: Konsolidierung des Holz-Erddammes.

Periode III: Bau eines Stein-Erddammes.

Periode IIIa-IIIb: Erhöhung des Stein-Erddammes und Bebauung

.

Datierung der Perioden I-VI

Erschließung der Flachwasserzone zur Anlage der Marktstätte ( Neuer Markt”) und eines neuen Hafens im 12. Jahrhundert. 


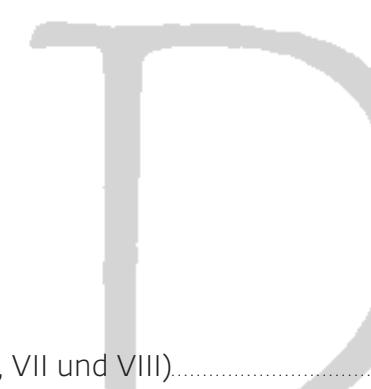

2.4 Zweiteilige Großbauten (Periode VI, VII und VIII)

$3 \quad$ Marktstätte nach dem Brand von 1398:

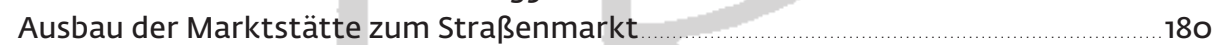

3.1 Einplanierung der Brandruine und Zuschüttung des Hafenbeckens .................................180

3.2 Metzig-Neubau, Brunnen und Entwässerungskanal auf der oberen Marktstätte..........180

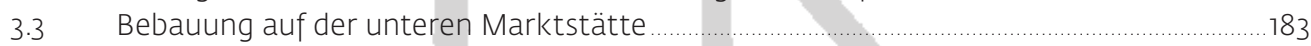

3.4 Entwicklung des hochmittelalterlichen Marktplatzes zum neuzeitlichen

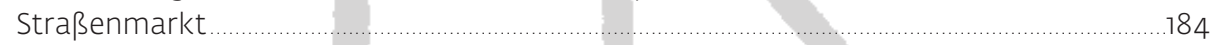

$4 \quad$ Zusammenfassung

4 KATALOG DER PERIODISIERTEN GRABUNGSBEFUNDE $\ldots . .188$

5 VERZEICHNISSE 247

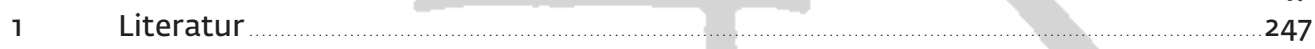

$2 \quad$ Bildnachweis 251

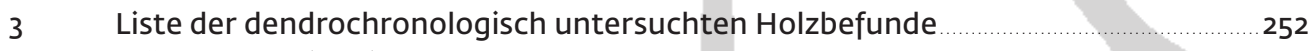

4 Münzenverzeichnis 255

$5 \quad$ Konkordanzliste der Grabungs-Befundnummern mit den periodisierten

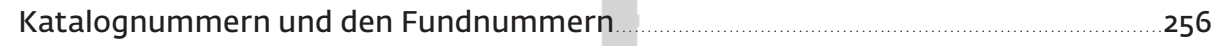

$6 \quad$ Verzeichnis der Tabellen $\quad$. 67

$7 \quad$ Verzeichnis der Beilagen $\quad 267$

DIE HOCH- UND SPÄTMITTELALTERLICHEN FUNDE VON DER KONSTANZER

MARKTSTÄTTE (Dorothee Ade unter Mitarbeit von Annika Hasler, Martin Rogier, Michael Seiz und Andreas Willmy) $\ldots 269$

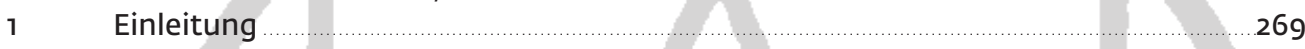

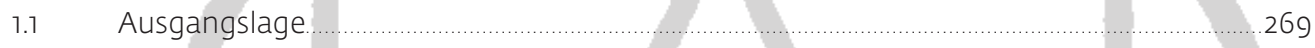

1.2 Bearbeitungsmethode

1.3 Anmerkungen zur Datierung und chronologischen Einordnung ….......................... 270

2 Gefäßkeramik 271

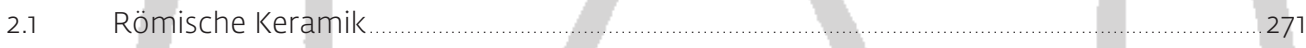

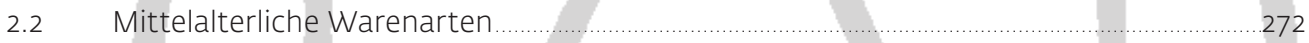

2.3 Obere westliche Marktstätte: Auffüllung der Flachwasserzone (Periode II-IIIb), Hausbebauung (Periode IV-VIIIa) und nach dem Brand (Periode IX) .............................276

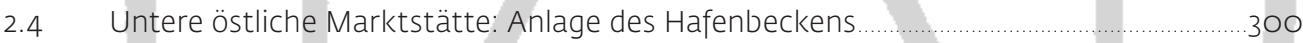

2.5 Brotlaube $\ldots \ldots \ldots \ldots$

$3 \quad$ Sonderfunde aus Keramik $\quad 311$

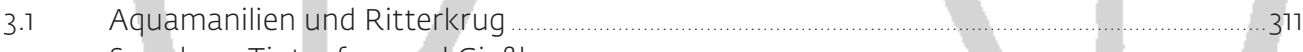

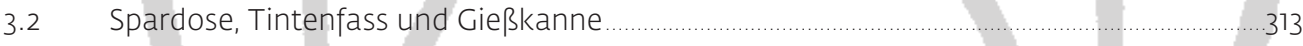

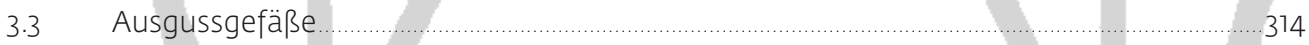

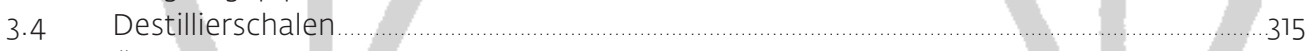

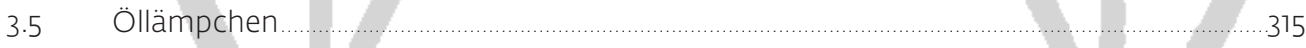

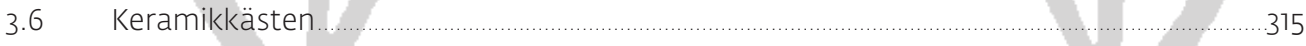

3.7 Töpfchen oder Tiegel ___ _ _ . 315

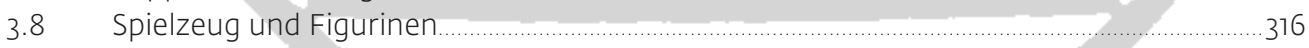

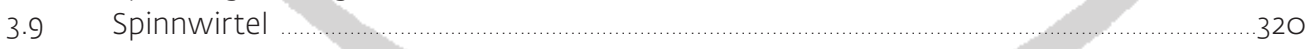

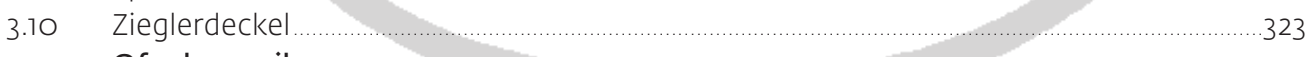

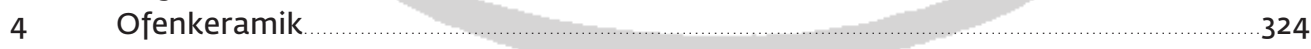

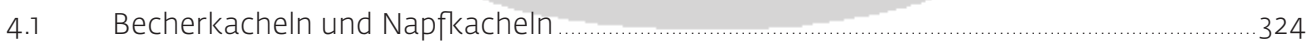

4.2 Gefäßkacheln mit eingesetztem Blatt, Pilzkachel................................................................329

4.3 Blatt-, Nischen- und Halbzylinderkacheln _....................................................................... 330

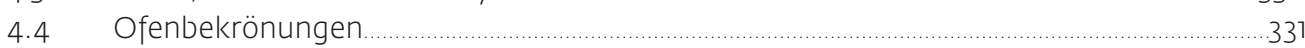

4.5 Zusammenfassende Betrachtungen zur Ofenkeramik ..............................................333 


\begin{tabular}{|c|c|c|}
\hline 5 & Baukeramik. & .334 \\
\hline 5.1 & Ziegel ................. & $\ldots 334$ \\
\hline 5.2 & Backsteine...... & 337 \\
\hline 5.3 & Bodenfliese $\quad 1 \quad \alpha$ & $\ldots 337$ \\
\hline 5.4 & Zusammenfassende Betrachtungen zur Baukeramik.... & 337 \\
\hline 6 & Glasfunde & 337 \\
\hline 6.1 & Hohlgläser ..... & 337 \\
\hline 6.2 & Flachgläser 1 & 343 \\
\hline 6.3 & Zusammenfassende Betrachtungen zu den Glasfunden.. & 345 \\
\hline 7 & Metallfunde & 345 \\
\hline 7.1 & Messer $\ldots-1-\cdots \cdots$ & 345 \\
\hline 7.2 & Waffen & 350 \\
\hline 7.3 & Pferde- und Reitzubehör...... & 351 \\
\hline 7.4 & Haus und Haushalt & .351 \\
\hline 7.5 & schmuck und Zierrat & 357 \\
\hline 7.6 & Freizeit, Schrift und Handel.... & 359 \\
\hline 7.7 & Produktion und Wirtschaft & 362 \\
\hline 8 & Funde aus Knochen und Geweih & 363 \\
\hline 8.1 & Würfel & 363 \\
\hline 8.2 & Knochenperlen und -ringe für Paternoster & 366 \\
\hline 8.3 & Artefakte und Abfälle . & 367 \\
\hline 9 & Holzfunde & 368 \\
\hline 9.1 & Dauben geböttcherter Gefäße & 368 \\
\hline 9.2 & Gedrechselte Teller, Schüsseln und Schalen.... & 373 \\
\hline 9.3 & Löffel & 374 \\
\hline 9.4 & Balken, Bretter und Verbindungselemente.... & 374 \\
\hline 9.5 & Sonstige Holzfunde & 374 \\
\hline 10 & Steinfunde & 375 \\
\hline 11 & Zusammenfassung . & 376 \\
\hline 17.1 & Periode II-IIIb: Auffüllung der Flachwasserzone (kurz vor 1130 bis 1165 )............. & 376 \\
\hline 11.2 & $\begin{array}{l}\text { Periode IV: Schwellrahmenbau auf Pfostenfundamenten (1165/66 bis zweites } \\
\text { Viertel des } 13 . J \text {. J.). }\end{array}$ & 377 \\
\hline 11.3 & PeriodeV: Kleinbau mit Fachwerkwänden (um 1250 bis nach Mitte 13. Jh.) & 378 \\
\hline 11.4 & $\begin{array}{l}\text { Periode IV-V: Nutzungsbereiche östlich und westlich des Schwellrahmenbaus } \\
\text { und Nutzungsablagerung im Hafenbecken (1165/66 bis nach Mitte } 13 . \text { Jh.) }\end{array}$ & 378 \\
\hline 11.5 & Periode VI: Schwellenbau und seeseitiger Steinbau, Nutzungsablagerung im & 270 \\
\hline 11.6 & $\begin{array}{l}\text { Periode VII: Zweiteiliger Großbau und Nutzungsablagerung im Hafenbecken } \\
\text { (um } 1270 \text { bis frühes 14. Jh.) }\end{array}$ & \\
\hline 11.7 & $\begin{array}{l}\text { Periode VIII: Seeseitiger Steinbau mit angebautem Schwellenbau } \\
\text { (nach } 1300 \text { bis 1398). }\end{array}$ & \\
\hline 11.8 & 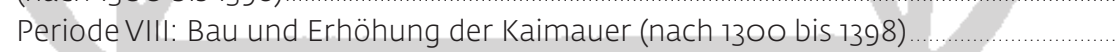 & 3.381 \\
\hline 11.9 & $\begin{array}{l}\text { Erste bis dritte Ablagerung: } \\
\text { Nutzungsablagerungen im Hafenbecken von Schnitt } 3 \text { und } 4 \text { (ca. 1250-1398).... }\end{array}$ & \\
\hline 11.10 & Periode IX: nach dem Brand 1398 & 383 \\
\hline 11.11 & $\begin{array}{l}\text { Brotlaube, Periode II-V: Holz-Erddamm, Stein-Erddamm, Ausbau und Nutzung } \\
\text { (nach Mitte 12.Jh. bis zweite Hälfte 13.Jh.) }\end{array}$ & \\
\hline 17.12 & Rückschau und Ausblick & 384 \\
\hline \multicolumn{3}{|c|}{ 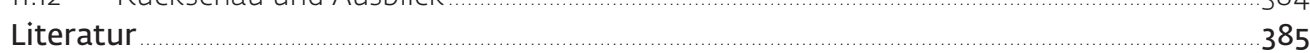 } \\
\hline Bildn & chweis... & 391 \\
\hline Fundl & atalog & \\
\hline
\end{tabular}




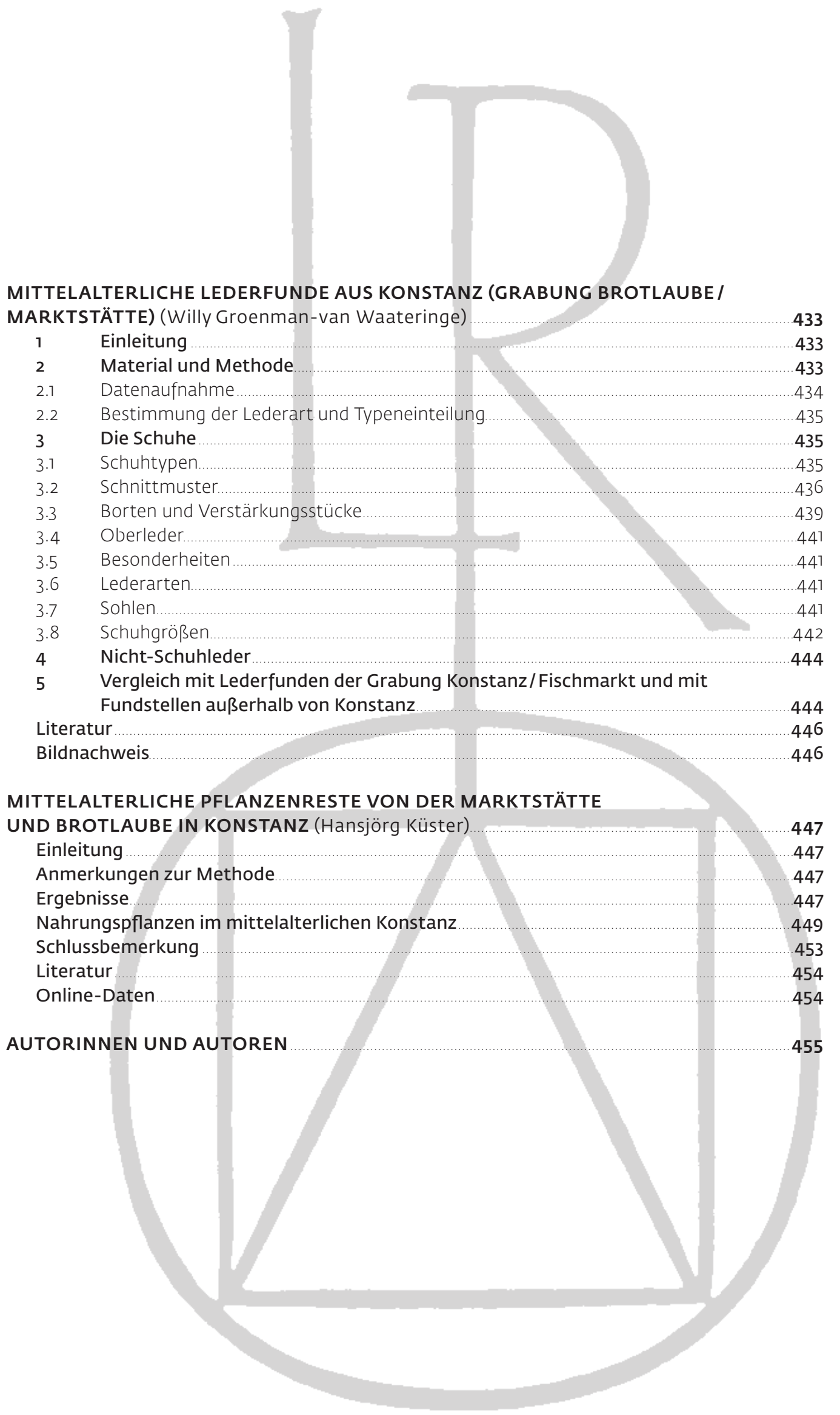

